\title{
Detection of arsenosugars from kelp extracts via IC-electrospray ionization-MS-MS and IC membrane hydride generation ICP-MS $\dagger$
}

\author{
Patricia A. Gallagher, Xinyi Wei, Jody A. Shoemaker, Carol A. Brockhoff and \\ John T. Creed* \\ US EPA NERL Microbiological and Chemical Exposure Assessment Research Division, \\ Cincinnati, $\mathrm{OH} 45268$, USA
}

Received 2nd August 1999, Accepted 21st September 1999

\begin{abstract}
The selectivity and the ability to obtain structural information from detection schemes used in arsenic speciation research are growing analytical requirements driven by the growing number of arsenicals extracted from natural products and the need to minimize misidentification in exposure assessments. Three arsenosugars were extracted from ribbon kelp utilizing accelerated solvent extraction. The three arsenosugars were separated from other arsenicals with near baseline resolution using a PRP-X100 column and a $20 \mathrm{~mm}\left(\mathrm{NH}_{4}\right)_{2} \mathrm{CO}_{3}$ mobile phase at a $\mathrm{pH}$ of 9 with IC-ICP-MS detection. Utilizing these chromatographic conditions, the molecular weight was determined for each arsenosugar utilizing ion chromatography-electrospray ionization-mass spectrometry (IC-ESI-MS) in the positive ion mode. The molecular weight and retention times for the three arsenicals are $328 \mathrm{u}(4.6 \mathrm{~min}), 482 \mathrm{u}(8.2 \mathrm{~min})$ and $392 \mathrm{u}(14.2 \mathrm{~min})$. The IC-ESI-MS-MS spectra from each of the arsenosugars were compared to the spectra reported in the literature, which were obtained via direct infusion of standard materials. All three MS-MS spectra contain $m / z$ 237, 195 and 97, which are fragments of the base dimethylarsinylriboside common to all the arsenosugars. Adequate sensitivity for each arsenical was achieved using a $6.1 \mathrm{ng}$ and a $22 \mathrm{ng}$ injection for IC-ESI-MS and IC-ESI-MS-MS, respectively. Given the unavailability of standards, the arsenosugar distribution was determined via relative chromatographic areas using IC-ICP-MS. The IC-ICP-MS indicated the presence of an arsenic heteroatom within the same retention windows in which the arsenosugars were detected via IC-ESI-MS. The IC-ESI-MS and IC-ESI-MS-MS detection scheme provided structural information but at reduced sensitivity. In an attempt to preserve sensitivity and improve selectivity of the IC-ICP-MS, an on-line membrane hydride generation detection scheme was evaluated. The hydride system indicated that the three unknown peaks (arsenosugars) were not hydride active, thereby simplifying the chromatographic resolution needed to quantitate the more toxicologically important arsenicals, such as MMA, DMA, As(III) and As(v), while minimizing the potential for misidentification.
\end{abstract}

\section{Introduction}

The two major pathways for arsenic exposure are drinking water and dietary intake. The geographical distribution of arsenic in surface water and ground water in the US has been estimated by Frey and Edwards. ${ }^{1}$ Inorganic arsenic is the predominant species present in drinking water and it is estimated that approximately $15 \%$ of the US population is exposed to arsenic in drinking water at concentrations greater than $2 \mu \mathrm{g} \mathrm{L}^{-1}$. Dietary exposure to total arsenic (arsenic determined after acid digestion) has been estimated. ${ }^{2-4}$ The naturally occurring and anthropogenic arsenicals are assimilated into many foods with the highest concentrations being found in fish and shellfish. Unlike water, the dietary sources of arsenic contain a variety of arsenicals. ${ }^{5-14}$ Some of these arsenicals are thought to be carcinogenic, others are thought to be cancer promoters, while some are considered non-toxic. This species-dependent toxicity of arsenicals requires analytical techniques capable of distinguishing the toxic from the nontoxic chemical forms. Therefore, an accurate risk assessment which incorporates the two major sources of arsenic exposure must estimate the toxic and non-toxic species found in the dietary components. One essential aspect of an accurate risk assessment for arsenic is sensitive and selective analytical

$\dagger$ US Government Copyright.

$\ddagger$ National Research Council Postdoctoral Fellow. methods capable of speciating arsenic in a variety of exposure assessment matrices.

The analytical capability to speciate arsenic in a wide variety of sample matrices is a rapidly growing research area. Analytical capability to speciate arsenic has been demonstrated in environmental matrices, ${ }^{15-19}$ biologicals ${ }^{20-32}$ and dietary samples ${ }^{5-14,33-36}$. Much of this research has focused on the use of atomic spectroscopy and more recently ICP-MS as the detector of choice because of the inherent sensitivity and elemental selectivity. The ability to obtain structural information from detection schemes used in arsenic speciation research is a growing analytical requirement driven by the growing number of arsenicals extracted from natural products and the need to minimize misidentification in exposure assessments. This growing list produces the potential for false positives based on insufficient chromatographic resolution or detector selectivity. Improved chromatographic resolution via capillary electrophoresis is an active area of research within arsenic speciation. ${ }^{37,38}$ Alternatively, the use of electrospray ionization (ESI) mass spectrometry as a detector for LC can provide structural information ${ }^{39,40}$ on eluting arsenicals and, in turn, improve detector selectivity. ${ }^{41-43}$ This structural information is obtained at the cost of reduced sensitivity relative to ICP-MS. A third approach to minimizing misidentification is to capitalize on the inability of highly derivatized arsenicals to produce a volatile hydride. ${ }^{44,45}$ This in effect improves the selectivity of atomic spectroscopy based detection systems while preserving the required sensitivity. This paper will report on the use of 
IC-ESI-MS-MS and IC hydride generation ICP-MS as a means of improving detector selectivity relative to conventional atomic spectroscopy. The IC-ESI-MS-MS system will be used to characterize three arsenosugars as they elute from a conventional anion exchange column while IC hydride generation ICP-MS will be used to preserve sensitivity and demonstrate an added degree of selectivity.

\section{Experimental}

\section{Reagents}

The ribbon kelp (Alaria marginata) was received from Puget Sound, WA, USA. The HPLC grade methanol $(\mathrm{MeOH})$ and trace metal grade ammonium hydroxide $\left(\mathrm{NH}_{4} \mathrm{OH}\right)$ originated from Fisher Scientific (Pittsburgh, PA, USA). The ACS reagent grade ammonium carbonate $\left[\left(\mathrm{NH}_{4}\right)_{2} \mathrm{CO}_{3}\right]$ was purchased from Aldrich (Milwaukee, WI, USA) while the ammonium phosphate $\left[\left(\mathrm{NH}_{4}\right)_{2} \mathrm{HPO}_{4}\right]$ and the ultrapure reagent grade nitric acid $\left(\mathrm{HNO}_{3}\right)$ were from J. T. Baker (Phillipsburg, NJ, USA). For hydride generation, trace metal grade $\mathrm{HCl}$ (Fisher, Pittsburgh, PA, USA) was used, and $\mathrm{NaBH}_{4}(97+\%$, Alfa AESAR, Johnson Matthey, Ward Hill, MA, USA) was prepared in $0.1 \mathrm{~m} \mathrm{NaOH}(97+\%$, certified ACS; Fisher, Fair Lawn, NJ, USA) solution for stabilization. $\mathrm{K}_{2} \mathrm{~S}_{2} \mathrm{O}_{8}(99+\%$, ACS reagent) was purchased from Aldrich. The water used was always $18 \mathrm{M} \Omega$ by Millipore (Bedford, MA, USA). The arsenite $[\mathrm{As}(\mathrm{III})]$ and arsenate [As(v)] were purchased from Spex CertiPrep (Metuchen, NJ, USA). Dimethylarsinic acid (DMA) and disodium methylarsenate (MMA) were from Chem Services (West Chester, PA, USA). Arsenobetaine (AsB) was from University of British Columbia, Department of Chemistry (Vancouver, Canada). All standard materials were prepared based on arsenic and standardized against NIST 1643c obtained from the US Department of Commerce National Institute of Standards and Technology (Gaithersburg, MD, USA).

\section{Sample preparation-accelerated solvent extraction}

The ribbon kelp was freeze dried utilizing a VirTis lyophilizer (Gardiner, NY, USA) followed by homogenization in an Osterizer blender (Milwaukee, WI, USA). The ribbon kelp samples were extracted using an ASE 200 accelerated solvent extractor system (Dionex, Sunnyvale, CA, USA). A specialized $3 \mathrm{~mL}$ ASE cell was utilized for this study. The ribbon kelp was mixed with Empore Filter Aid 400 high density glass beads from Varian (Harbor City, CA, USA). The glass beads were used as a support medium and prevented the ASE cell from clogging due to the ribbon kelp expanding. The ASE parameters were: $30+70 \mathrm{MeOH}-\mathrm{H}_{2} \mathrm{O}, 500 \mathrm{psi}$, ambient temperature, $1 \mathrm{~min}$ heat step, $1 \mathrm{~min}$ static step, $30 \%$ flush step, 1 cycle and a $120 \mathrm{~s}$ purge. The Zymark TurboVap LV evaporator was set to $50^{\circ} \mathrm{C}$ with a nitrogen purge. The extract was next brought to dryness. The residue was then redissolved in water and treated with a maxi clean $\mathrm{C}_{18}$ cartridge $(900 \mathrm{mg})$ from Alltech Associates, Inc. (Deerfield, IL, USA). The $\mathrm{C}_{18}$ purified sample was used in all further testing. A summary of the ASE instrumental conditions can be found in Table 1.

\section{Chromatography ICP-MS and ESI-MS-MS}

The ion chromatography was completed utilizing a Dionex Gradient pump (Model GPM2), which utilizes a pre- and postcolumn six-way valve injector. The post-column injection introduces the marker peak and the pre-column injection is used for chromatographic separation. Three different chromatographic separations were utilized. The first chromatographic separation conditions consist of an ION 120 column from Interaction Chromatography (San Jose, CA, USA) with a $40 \mathrm{~mm}\left(\mathrm{NH}_{4}\right)_{2} \mathrm{CO}_{3}, \mathrm{pH} 10.5$ mobile phase. The flow rate was

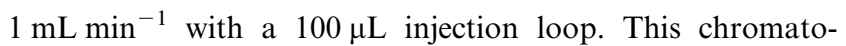
graphic separation was used with direct nebulization ICP-MS detection. The second chromatographic separation conditions involve a PRP-X100 column from Hamilton (Reno, NV, USA) and a $20 \mathrm{~mm}\left(\mathrm{NH}_{4}\right)_{2} \mathrm{CO}_{3}, \mathrm{pH} 9.0$ mobile phase. The flow rate

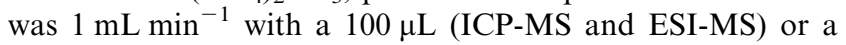
$360 \mu \mathrm{L}$ (ESI-MS-MS) injection loop. This chromatographic separation was used with direct nebulization ICP-MS and ESIMS-MS detection. The third chromatographic separation used in the hydride research incorporates the Hamilton PRP-X100 column with a $14 \mathrm{~mm}\left(\mathrm{NH}_{4}\right)_{2} \mathrm{HPO}_{4}-14 \mathrm{~mm} \mathrm{HNO}_{3}, \mathrm{pH} 6.0$, mobile phase. The flow rate was $1 \mathrm{~mL} \mathrm{~min}^{-1}$ with a $50 \mu \mathrm{L}$ injection loop. A summary of the chromatographic conditions can be found in Table 1 .

\section{Direct nebulization and membrane hydride ICP-MS detection}

The ICP-MS used for direct nebulization was a Plasma Quad 3 from VG Elemental (Franklin, MA, USA). The flow rates for the plasma, auxiliary and nebulizer were $13.0 \mathrm{~L} \mathrm{~min}^{-1}$, $0.80 \mathrm{~L} \mathrm{~min}^{-1}$ and $0.68 \mathrm{~L} \mathrm{~min}^{-1}$, respectively, with a forward power of $1350 \mathrm{~W}$. Data collection was completed using single ion monitoring of $\mathrm{m} / \mathrm{z} 75$.

The hydride generation ICP-MS instrument was a HewlettPackard (Avondale, PA, USA) 4500 series. The membrane hydride generation system has been described previously. ${ }^{18}$ This system has been modified to include the on-line postcolumn photo-oxidation process to allow for the detection of highly derivatized arsenicals. The on-line photo-reactor PHRED was purchased from Aura Industries, Inc. (State Island, NY, USA). The reactor compartment was equipped with an $8 \mathrm{~W}$ UV lamp $(254 \mathrm{~nm})$ and a polished stainless steel support plate with holes at the bottom, which allowed the Teflon tubing reaction coil to extend in or out of the reactor. For better irradiation efficiency, the original flat-shaped PHRED knitted reaction coil (designed to fit on the bottom support plate under the UV lamp) was replaced with a $5 \mathrm{~m}$ thin-wall microbore PTFE tubing (id $0.56 \mathrm{~mm}$, wall $0.02 \mathrm{~mm}$, Cole-Parmer Instrument Company, Vernon Hills, IL, USA) reactor, which was braided around the UV lamp. The IC effluent was mixed with $2 \% \mathrm{~K}_{2} \mathrm{~S}_{2} \mathrm{O}_{8}$ solution at a three-way PTFE manifold mixer and passed through the reaction coil under UV irradiation. A cooling device for the UV lamp was not provided with PHRED, and for this reason a warm up period of $15-20 \mathrm{~min}$ was used to ensure that thermal equilibrium was achieved.

\section{ESI-MS-MS}

The Finnigan MAT TSQ 700 (San Jose, CA, USA) triple quadrupole mass spectrometer, equipped with an API I interface, was utilized in the ESI-MS and ESI-MS-MS mode. The instrument was initially tuned for ESI using a myoglobinMRFA mixture. The electrospray needle was held at $5 \mathrm{kV}$ and was operated in the positive ion mode. The heated capillary $\left(275^{\circ} \mathrm{C}\right)$, the nitrogen sheath gas $(80 \mathrm{psi})$ and the nitrogen auxiliary gas (35) were optimized using arsenobetaine infused at $1 \mathrm{~mL} \mathrm{~min}{ }^{-1}$ in $20 \mathrm{~mm}\left(\mathrm{NH}_{4}\right)_{2} \mathrm{CO}_{3}$. The heated capillary and manifold temperatures were 275 and $100{ }^{\circ} \mathrm{C}$, respectively. In MS experiments, the instrument was scanned from 130 to $550 \mathrm{u} \mathrm{s}^{-1}$. The MS-MS experiments were conducted using a collision energy of $30 \mathrm{eV}$ and an argon pressure of 1 mTorr. The IC-ESI-MS-MS experiment was performed as follows: during retention time windows of $0-6 \mathrm{~min}, 6-10 \mathrm{~min}$ and 10 $16 \mathrm{~min}, \mathrm{Q}_{1}$ was set to pass $m / z 329,483$ and 393 , respectively, and $\mathrm{Q}_{3}$ was scanned at 75-331, 75-485 and 75-395 u s${ }^{-1}$, respectively. A summary of these instrumental conditions can be found in Table 1 . 
Table 1 Summary of experimental conditions

Chromatography

PRP-X100 column

Direct analysis: $20 \mathrm{~mm}\left(\mathrm{NH}_{4}\right)_{2} \mathrm{CO}_{3}, \mathrm{pH} 9.0$

Hydride analysis: $14 \mathrm{~mm}\left(\mathrm{NH}_{4}\right)_{2} \mathrm{HPO}_{4}-14 \mathrm{~mm} \mathrm{HNO}_{3}, \mathrm{pH} 6.0$

ION 120 column

Direct analysis: $40 \mathrm{~mm}\left(\mathrm{NH}_{4}\right)_{2} \mathrm{CO}_{3}, \mathrm{pH} 10.5$

Flow rate and injection volume

ICP-MS and ESI-MS: $1 \mathrm{~mL} \min ^{-1}$ and $100 \mu \mathrm{L}$

Hydride analysis ICP-MS: $1 \mathrm{~mL} \mathrm{~min}^{-1}$ and $50 \mu \mathrm{L}$

ESI-MS/MS analysis: $1 \mathrm{~mL} \mathrm{~min}^{-1}$ and $360 \mu \mathrm{L}$

Accelerated solvent extraction-

Solvent: $30+70(\mathrm{w} / \mathrm{w}) \mathrm{MeOH}-\mathrm{H}_{2} \mathrm{O}$

Pressure: 500 psi

Temperature: ambient

Flush percentage: $30 \%$

Cell size: $3 \mathrm{~mL}$

Static time: $1 \mathrm{~min}$

Purge time: $120 \mathrm{~s}$

\section{Hydride generation-}

$\mathrm{NaBH}_{4}: 1.5 \%(\mathrm{w} / \mathrm{w})$ in $0.1 \mathrm{~m} \mathrm{NaOH}$

$\mathrm{HCl}: 35 \%(\mathrm{w} / \mathrm{w})$

Potassium persulfate: $2 \%(\mathrm{w} / \mathrm{w})$

Membrane: expanded polytetrafluoroethylene microporous tubing

Electrospray MS and electrospray MS-MS-

Finnigan MAT TSQ 700

Auxiliary flow: 35 nitrogen (arbitrary units)

Sheath flow: 80 psi nitrogen

Capillary temperature: $275^{\circ} \mathrm{C}$

Electrospray needle potential: $5 \mathrm{kV}$

\section{Results and discussion}

Edmonds and Francesconi ${ }^{46}$ characterized 2 arsenosugars extracted from brown kelp using IR and NMR in 1981. Corr and Larsen ${ }^{41}$ characterized three arsenosugars via ESI-MS-MS using direct infusion for sample introduction. These three arsenosugars are retained on the ION $120^{47}$ and PRP-X10047 anion exchange columns using typical mobile phases $\left[\left(\mathrm{NH}_{4}\right)_{2} \mathrm{CO}_{3}\right]$ and $\mathrm{pHs}(>\mathrm{pH} 9)$. Given these chromatographic conditions, the arsenosugars can potentially co-elute with As(III), DMA and AsB producing a false positive or misidentification. Fig. 1 demonstrates this co-elution problem utilizing the ION 120 column, $40 \mathrm{~mm}\left(\mathrm{NH}_{4}\right)_{2} \mathrm{CO}_{3}$ at $\mathrm{pH} 10.5$.

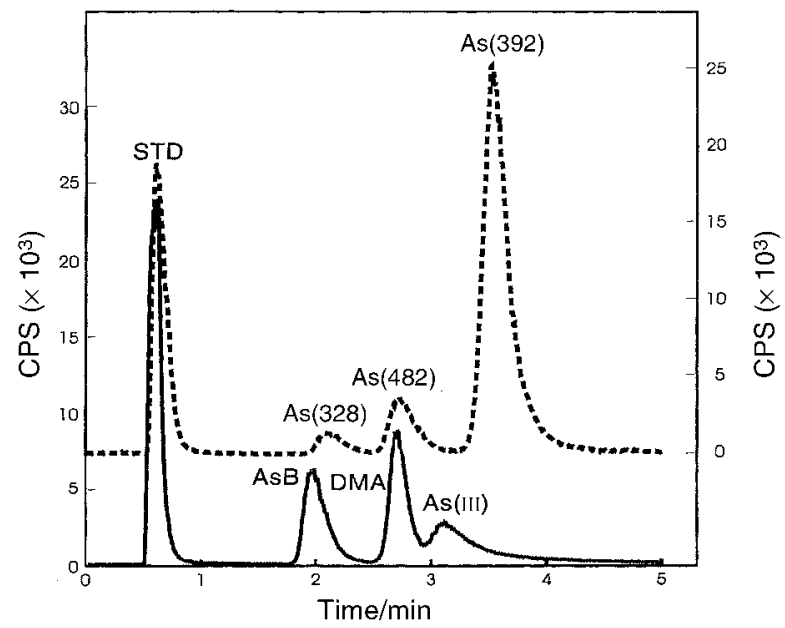

Fig. 1 Potential for misidentification of arsenicals using IC-ICP-MS detection. Chromatographic conditions: ION 120 column; isocratic $40 \mathrm{~mm}\left(\mathrm{NH}_{4}\right)_{2} \mathrm{CO}_{3}, \mathrm{pH} 10.5,1 \mathrm{~mL} \mathrm{~min}^{-1}$. Solid line, $1 \mathrm{ng}$ of each arsenical; broken line, $6.1 \mathrm{ng}$ arsenosugars based on total As measurement. Numbers in parentheses refer to molecular weights established by ESI-MS.
These conditions are standard conditions used in our laboratory for arsenic speciation in seafood extracts. Fig. 1 contains two chromatograms collected using these separation conditions and direct nebulization ICP-MS detection. The first chromatogram (top, broken line, $y$ axis on right of graph) is an injection of a kelp extract which contained three arsenosugars (combined $6.1 \mathrm{ng}$ injected, based on a total arsenic measurement). See Table 2 for arsenical structures. The second chromatogram (bottom, solid line, $y$ axis on left of graph) is a standard injection of $1.0 \mathrm{ng}$ of each of AsB, DMA and As(III) These two chromatograms clearly indicate the potential for misidentification of arsenosugar 482 (482 is based on molecular mass, see IC-ESI-MS section and Table 2) as DMA and arsenosugar 328 as AsB. Similar chromatograms are obtained if a PRP-X100 column is used. ${ }^{47}$ Le et al. ${ }^{48}$ have reported similar co-elution and potential misidentification effects (on anion exchange columns) in human urine produced by the ingestion of arsenosugars from seafoods. Le et al. ${ }^{44}$ utilized ICP-MS and atomic fluorescence as detectors and relied on elution times for identification. In order to minimize the potential for misidentification, a separation using a PRP-X100 column and $20 \mathrm{~mm}\left(\mathrm{NH}_{4}\right)_{2} \mathrm{CO}_{3}$ mobile phase at pH 9.0 was developed. This produces a near baseline separation of all known anionic arsenicals in $30 \mathrm{~min}$. This separation allowed the retention times obtained for the three arsenosugars using IC-ICP-MS to be used to verify the retention times of the $[\mathrm{M}+\mathrm{H}]^{+}$species found utilizing IC-ESI-MS. The relative abundance (based on chromatographic peak areas) of the three arsenosugars in the kelp extract determined by IC-ICP-MS was $5.0 \%$ (arsenosugar 328, $\mathrm{m} / \mathrm{z} 329$ ), 11.0\% (arsenosugar 482 , $\mathrm{m} / \mathrm{z} 483$ ) and $84.0 \%$ (arsenosugar 392, $\mathrm{m} / \mathrm{z} 393$ ). This distribution was calculated using the extraction conditions in Table 1 and an extraction efficiency of $55 \%$ (i.e., the kelp after extraction contained $45 \%$ of the available arsenic based on a total arsenic digestion).

\section{IC-ESI-MS and MS-MS of arsenosugars from ribbon kelp}

Electrospray ionization coupled with IC-MS and IC-MS-MS was utilized to determine the identity of the three unknown arsenic peaks (from a ribbon kelp extract) observed in Fig. 1. The chromatographic conditions were optimized (as mentioned above) to produce near baseline resolution of all arsenicals. The separation and detection conditions used in Fig. 2 and 3(a-c) are listed in Table 1. Fig. 2 is a chromatogram of the ribbon kelp extract obtained by positive ion IC-ESI-MS. The figure has three overlapping traces of $m / z 329\left([\mathrm{M}+\mathrm{H}]^{+}\right.$, arsenosugar $328), \quad m / z 483\left([\mathrm{M}+\mathrm{H}]^{+}\right.$, arsenosugar 482) and $\mathrm{m} / \mathrm{z} 393$ $\left([\mathrm{M}+\mathrm{H}]^{+}\right.$, arsenosugar 392). Except for arsenosugar 392, the signal at these masses is a minor component of the total ion current. The retention times for the three unknown peaks via IC-ESI-MS are within 0,4 and $7 \%$ (respectively) of the retention times obtained via IC-ICP-MS. This slight shift in retention times for late eluting peaks may be produced by the relatively weak mobile phase in combination with the $1: 10$ dilution factor used in the IC-ICP-MS analysis. The net effect is that the matrix anions in the IC-ESI-MS analysis are depleting the available sites (relative to IC-ICP-MS analysis) on the anion PRP-X100, thereby producing shorter retention times. From the ESI mass spectra obtained at these retention times, it was determined that the unknown arsenic peaks had molecular weights of 328 (arsenosugar 328), 482 (arsenosugar 482), and 392 (arsenosugar 392). These molecular weights matched molecular weights published by Corr and $\operatorname{Larsen}^{41}$ for the arsenosugars. This molecular weight match in combination with the confirmation of the presence of an arsenic heteroatom (via IC-ICP-MS) made the three arsenosugars a logical choice for tentative identification.

Collisionally activated decomposition (CAD) was performed on the $[\mathrm{M}+\mathrm{H}]^{+}$of each unknown arsenic peak to further 
Table 2 Arsenical chemical structures

$$
\text { Arsenious Acid }
$$
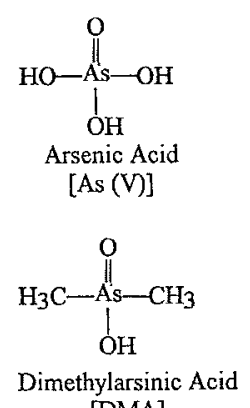

confirm that the unknown peaks were arsenosugars. To obtain adequate sensitivity in the MS-MS mode it was necessary to inject $360 \mu \mathrm{L}$ of an undiluted extract $(22.1 \mathrm{ng})$. The MS-MS

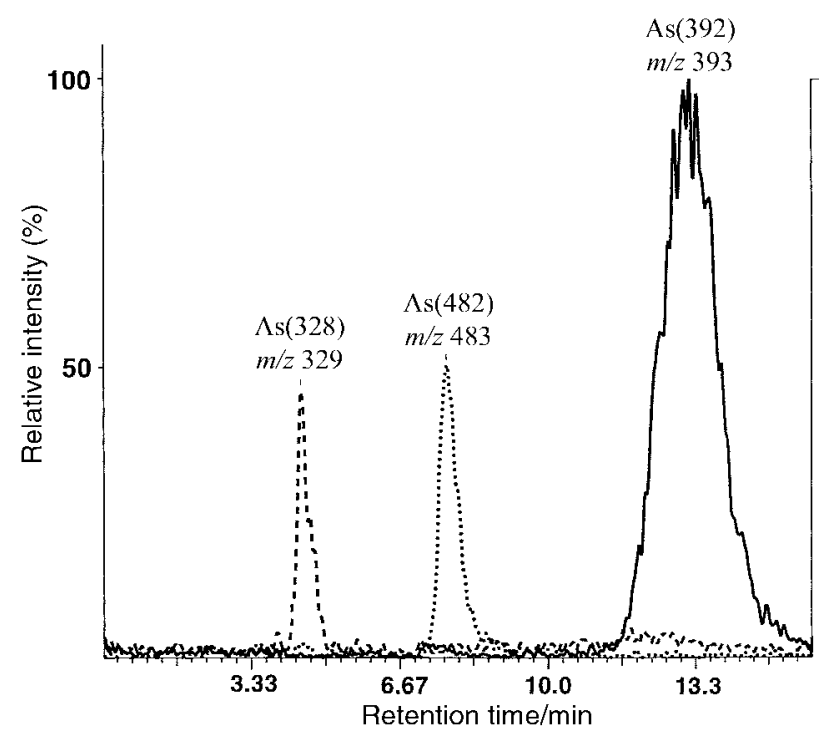

Fig. 2 Electrospray IC-MS mass chromatogram of arsenicals extracted from ribbon kelp. Chromatographic conditions: PRP-X100 column, isocratic $20 \mathrm{~mm}\left(\mathrm{NH}_{4}\right)_{2} \mathrm{CO}_{3}, \mathrm{pH}=9.0,1 \mathrm{~mL} \mathrm{~min}{ }^{-1}$. Broken line, $m / z$ 329; dotted line, $m / z$ 483; solid line, $m / z 393$. mass spectra obtained on the eluting peaks are shown in Fig. 3(a-c). The MS-MS spectra of the three unknown peaks (arsenosugar 328; arsenosugar 482; arsenosugar 392) are similar to the MS-MS spectra of the arsenosugars published by Corr and Larsen ${ }^{41}$, given the differences in the instrumentation used in the experiments. All three MS-MS spectra contain $\mathrm{m} / \mathrm{z} 237,195$ and 97, which are fragments of the base dimethylarsinylriboside, common to all the arsenosugars. While $\mathrm{m} / \mathrm{z} 97$ could be $\mathrm{OSO}_{3}{ }^{+}$in arsenosugar 392, this would not explain its presence in arsenosugar 328 or arsenosugar 482. Thus, the structure shown in Fig. 3(a-c) involving the pentose moiety common to all the sugars is one possible assignment for $\mathrm{m} / \mathrm{z} 97$ in all three arsenosugars. This assignment is in agreement with Pergantis et al. ${ }^{39}$ In the case of arsenosugar 392, some of the $\mathrm{m} / \mathrm{z} 97$ signal in the MS-MS spectrum may be due to $\mathrm{OSO}_{3}{ }^{+}$. The MS-MS spectrum of arsenosugar 482 shows additional fragments at $\mathrm{m} / \mathrm{z} 465$ (loss of water), 391 and 329, which are fragments of the phosphate functional group. The MS-MS spectrum of arsenosugar 392 does not contain the daughter ions at $m / z 149,167$ and 279 reported by Corr and Larsen. ${ }^{41}$ One possibility for this difference is that Corr and Larsen ${ }^{41}$ noted large phthalate contamination in their arsenosugars, which dominated the MS spectra. If this contamination was extremely severe, it is

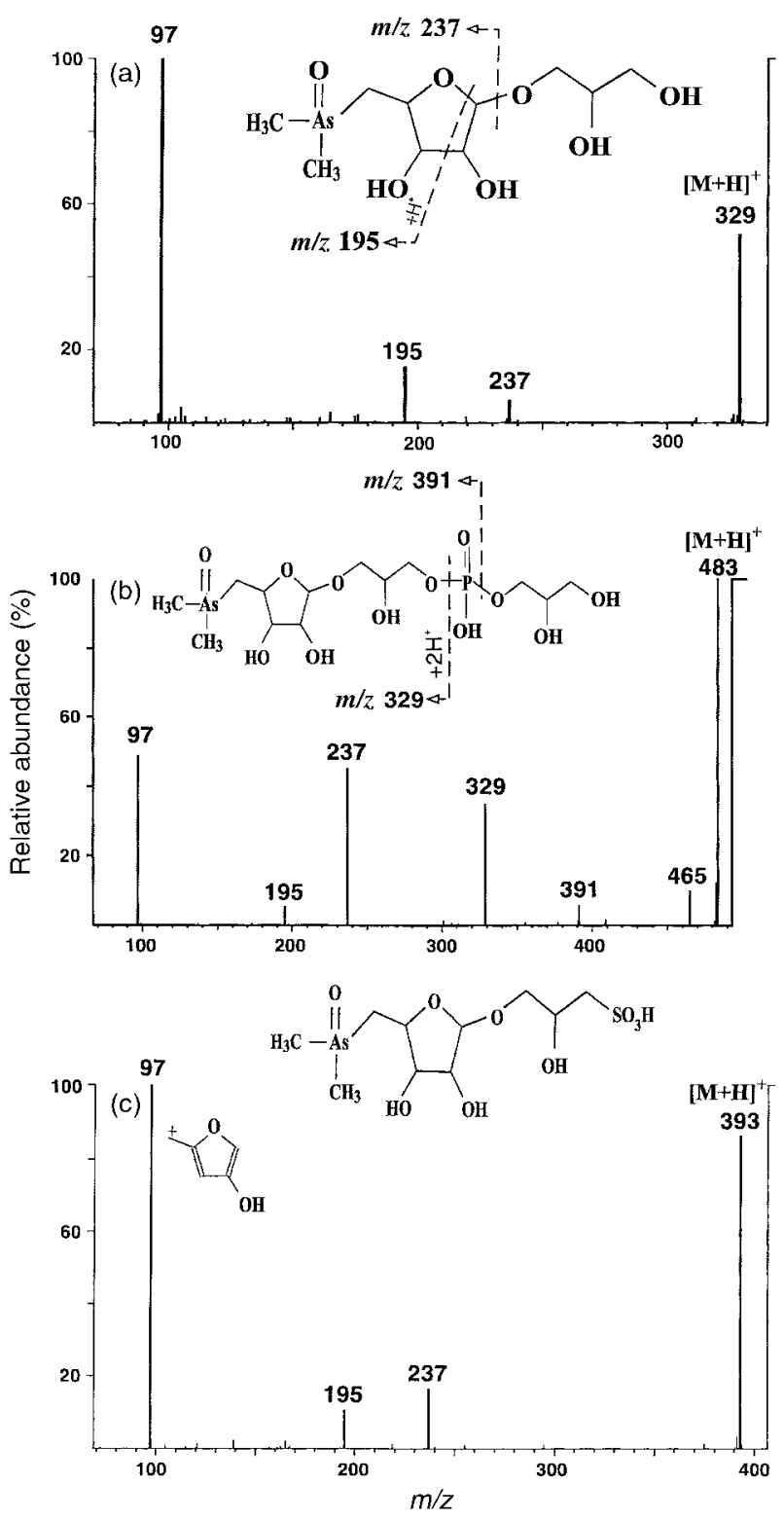

Fig. 3 MS-MS spectra of three arsenosugars. (a) MS-MS of As (328); (b) MS-MS of As (482); (c) MS-MS of As (392). 
possible that the daughter ions at $\mathrm{m} / \mathrm{z} 149,167$ and 279 observed during direct infusion were produced by dioctylphthalate (nominal MW 390) via carry over.

The MS-MS spectra shown in Fig. 3(a-c) provide additional data which indicate that the unknown chromatographic peaks are arsenosugars rather than the typical arsenicals found in the environment. These IC-ESI-MS and IC-ESI-MS-MS spectra, coupled with the retention time verification with ICP-MS, give credence to the presence of the three arsenicals but without arsenosugar standards this confirmation is tentative.

\section{Ion chromatography membrane hydride generation ICP-MS}

The arsenicals commonly used to assess exposure are inorganic arsenic [As(III) and As(v)], MMA and DMA. Therefore, an arsenic exposure assessment methodology should provide interference free detection of these species and minimize the potential for false positives from other arsenicals. This added degree of detector selectivity can be achieved by using a membrane hydride ICP-MS methodology without a postcolumn photo-oxidation step. A summary of these chromatographic and hydride generation conditions can be found in Table 1 . The highly substituted arsenicals (arsenobetaine, etc.) are not hydride active without photo-oxidation. ${ }^{44,45}$ This allows the highly substituted arsenicals to chromatographically co-elute with As(III), As(v), MMA or DMA without producing a false positive. This selectivity is demonstrated in Fig. 4(a-b).

Fig. 4(a) contains two chromatograms collected with the hydride generation system operating with a post-column on-

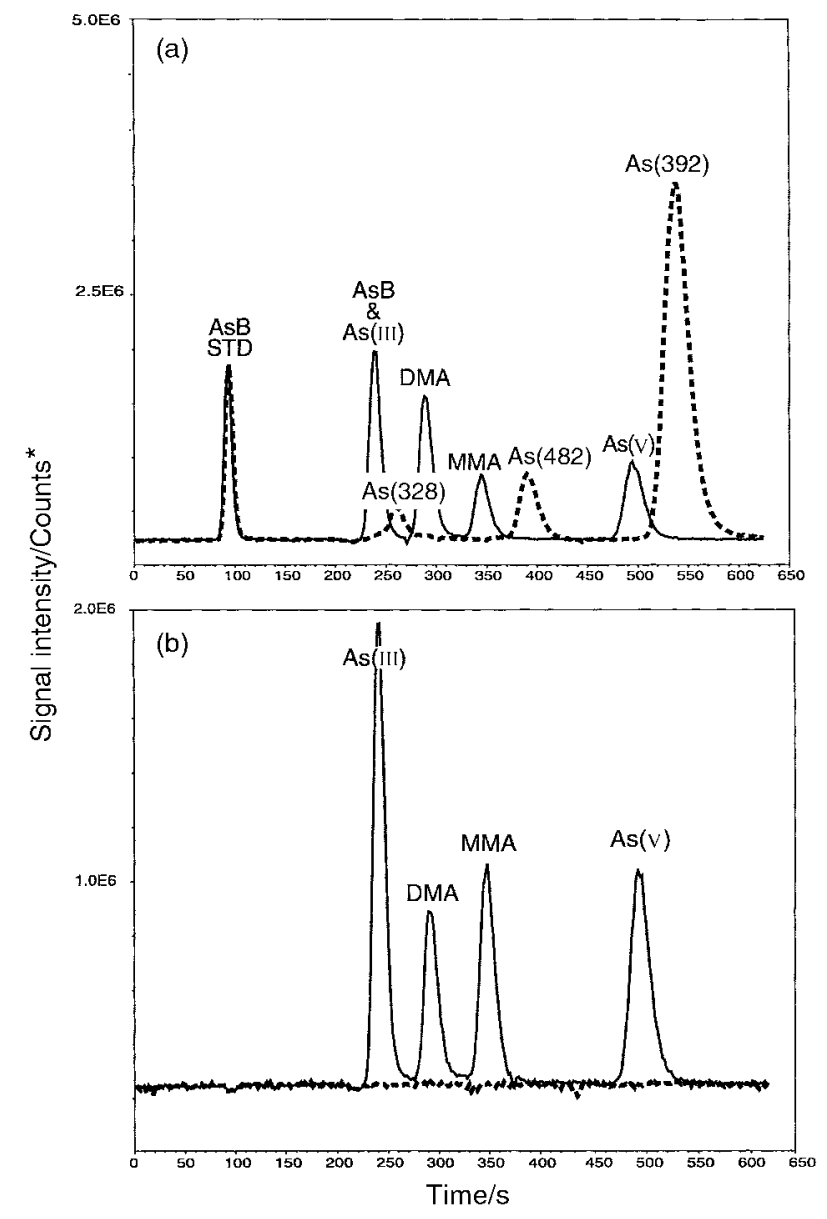

Fig. 4 Chromatographic separation of arsenicals using hydride generation with (a) on-line photo-oxidation. Chromatographic conditions: PRP-X100 column, isocratic $14 \mathrm{~mm}\left(\mathrm{NH}_{4}\right)_{2} \mathrm{HPO}_{4}-14 \mathrm{~mm} \mathrm{HNO}_{3}$, pH 6.0, $1 \mathrm{~mL} \mathrm{~min}^{-1}$. Solid line, $100 \mathrm{pg}$ DMA and As ${ }^{\mathrm{V}}, 50 \mathrm{pg} \mathrm{AsB}, \mathrm{As}^{3 \mathrm{III}}$ and MMA; broken line, $615 \mathrm{pg}$ of total arsenosugars injected. Numbers in parentheses refer to molecular weights established by ESI-MS. (b) Without on-line photo-oxidation. *Dwell time is $2 \mathrm{~s}$. line photo-oxidation step prior to detection. The separation conditions are dramatically different from those used in Fig. 13. The column is a PRP-X100 with a $14 \mathrm{~mm}\left(\mathrm{NH}_{4}\right)_{2} \mathrm{HPO}_{4}$ $14 \mathrm{~mm} \mathrm{HNO}_{3}, \mathrm{pH}$ 6.0, mobile phase. This mobile phase is used because it does not produce a gaseous species (i.e., $\mathrm{CO}_{2}$ from an ammonium carbonate mobile phase) within the hydride generation reaction and for this reason produces a more reproducible/steady baseline. The first chromatogram in Fig. 4(a) (indicated by the solid line) is an injection of $50 \mathrm{pg}$ of AsB, As(III), MMA and $100 \mathrm{pg}$ of DMA and As(v). The arsenobetaine peak marked AsB/STD is a post-column injection. This post-column injection of AsB indicates the on-line photo-oxidation step is functioning. Similar selectivity has been demonstrated by Le et al. ${ }^{44,48}$ using on-line microwave HGAAS. This chromatogram indicates a clean separation of the four arsenicals in approximately $9 \mathrm{~min}$. The second chromatogram (indicated by a broken line) in Fig. 4(a) is the separation of the ribbon kelp extract ( $0.6 \mathrm{ng}$ arsenosugar injected, based on a total measurement) achieved using the exact same chromatographic/hydride condition. All three arsenosugars are hydride active after being subjected to the on-line photo-oxidation process. In addition, the elution order is the same as in Fig. 1 and 2 and all three species still maintain a retention characteristic on the PRP-X100 column at a pH of 6.0. The combination of both chromatograms clearly indicates that the three arsenosugars do not produce a direct chromatographic overlap but do complicate the identification process based on retention time.

Fig. 4(b) contains two chromatograms in which the on-line photo-oxidation step is not utilized. The first chromatogram (indicated by a solid line) is a standard mix [100 pg As(v), DMA and $50 \mathrm{pg}$ of AsB, As(III) and MMA]. This clearly indicates the excellent sensitivity achievable using a hydride ICP-MS system. Note: The post-column injection of AsB does not produce a response. This indicates the photo-oxidation step is inoperable. The second chromatogram (indicated by a broken line) is a $50 \mu \mathrm{L}$ injection of the ribbon kelp extract (12.3 $\mathrm{ng} \mathrm{g}^{-1}$ arsenosugar based on a total measurement) without photo-oxidation. The flat baseline indicates that the arsenosugars in the ribbon kelp extract shown in Fig. 4(a) are not hydride active without some type of oxidative step prior to detection. Le et al. ${ }^{44}$ have demonstrated this via microwave digestion of arsenosugars in urine samples. The highly substituted arsenicals, e.g., AsB, arsenocholine (AsC), etc., are normally not hydride active without photo-oxidation. Arsenosugars produce non-volatile hydrides which are not detectable. Therefore, by carefully monitoring experimental conditions, highly substituted arsenicals could be co-eluted with As(III), As(v), MMA or DMA and detected without producing a false positive. These data provide additional evidence to indicate the presence of arsenosugars and that these arsenosugars [characterized by IC-ESI-MS-MS in Fig. 3(a-c)] are the only detected arsenicals in the ribbon kelp. In addition, the hydride generation mode of detection without the photooxidation step significantly simplifies the chromatograms while photo-oxidation provides the analyst with the flexibility of detecting the arsenosugars for exposure source information.

The above data clearly indicate the selectivity differences between IC-ESI-MS, IC-ICP-MS and IC-hydride-ICPMS. Selectivity is one aspect which is a growing requirement in arsenic speciation methodologies while instrumental sensitivity is challenged, especially in solid sample extracts. In an attempt to make a sensitivity comparison between IC-ICP-MS, IC-ESI-MS and IC-hydride-ICP-MS, a concentration for the $\mathrm{m} / \mathrm{z} 483$ peak was calculated from the relative area distribution and the total arsenic concentration in the extract. The relative area per cent of the $m / z 483$ peak is $11 \%$ and the total arsenic concentration in the extract is $613 \mathrm{ng} \mathrm{g}^{-1}$, therefore the $\mathrm{m} / \mathrm{z} 483$ peak is $67 \mathrm{ng} \mathrm{g}^{-1}$. Using $67 \mathrm{ng} \mathrm{g}^{-1}$ of arsenic as the concentration for the $\mathrm{m} / \mathrm{z} 482$ peak and calculating a 3 sigma 
detection limit based on peak height, the following detection limits were calculated. The 3 sigma detection limits were 4.5, 150 and $1.5 \mathrm{pg}$ for the IC-ICP-MS, IC-ESI-MS, and IChydride-ICP-MS, respectively. It should be noted that the ICESI-MS data collection and ESI experimental parameters were not optimized for detection limits but rather were compromised to facilitate the IC separation developed for IC-ICP-MS.

\section{Conclusions}

The elemental selectivity of atomic spectrometric detection provides excellent sensitivities relative to IC-ESI-MS but does not provide structural information available with IC-ESI-MS and IC-ESI-MS-MS. Hydride IC-ICP-MS provides unparalleled sensitivity and increased selectivity but still does not provide a structural identification capability for unknowns. Unlike ICP-MS the total ion current chromatograms obtained from an IC-ESI-MS-MS indicate a wide variety of co-extracted organic constituents eluting from the IC. This makes the identification of species which may contain arsenic very difficult without the use of the elemental information obtained via IC-ICP-MS. The arsenicals are, in effect, minor constituents via IC-ESI-MS while the ICP-MS can provide the elemental information necessary to identify IC retention windows of interest for the identification of arsenosugars from the ribbon kelp extract. The IC-ESI-MS spectra clearly indicate that the eluting species have the same molecular ion as those reported in the literature while the IC-ESI-MS-MS spectra confirm structural similarities to those reported in the literature. Finally, the ICP-MS chromatograms indicate the presence of an arsenic heteroatom during each of the three corresponding retention windows. From this perspective and a methods development standpoint the techniques are complementary and each provide supporting structural or elemental confirmation. Clearly, ICP-MS elemental information is inadequate in providing the structural information needed to identify these arsenosugars; on the other hand, IC-ESI-MS and IC-ESI-MSMS as applied in this paper lack the sensitivity necessary for arsenic exposure assessment. Hydride ICP-MS does provide an added degree of selectivity, which minimizes the required chromatographic resolution while providing an analytical response for the most toxicologically relevant arsenicals. From this perspective, the IC-ESI-MS-MS structural information has allowed the arsenosugars to be identified and this identification has allowed a hydride IC-ICP-MS analytical approach to be tested for false positives produced from arsenosugars.

\section{Acknowledgements}

The authors would like to thank Roseanne M. Lorenzana, US EPA Region 10, for her generosity in supplying us with the ribbon kelp sample utilized in this study. We would also like to recognize Douglas T. Heitkemper, US FDA, whose help through a cooperative study has been invaluable.

\section{References}

1 M. M. Frey and M. A. Edwards, J. Am. Water Works Assoc., 1997, 89, 105.

2 R. W. Dabeka, A. D. McKenzie, G. M. A. Lacroix, C. Cleroux, S. Bowe, R. A. Graham, H. B. S. Conacher and P. Verdier, J. AOAC Int., 1993, 76, 14.

3 E. L. Gunderson, J. AOAC Int., 1995, 78, 910.

4 E. L. Gunderson, J. AOAC Int., 1995, 78, 1353

5 J. S. Edmonds and K. A. Francesconi, Mar. Pollut. Bull., 1993, 26, 665 .

6 S. Branch, L. Ebdon and P. O’Neill, J. Anal. At. Spectrom., 1994, 9, 33 .
7 J. Alberti, R. Rubio and G. Rauret, Fresenius' J. Anal. Chem. 1995, 351, 420.

8 D. Velez, N. Ybanez and R. Montoro, J. Agric. Food Chem., 1995, 43, 1289 .

9 D. Velez, N. Ybanez and R. Montoro, J. Agric. Food Chem., 1996, 44, 859 .

10 T. Kaise, H. Yamauchi, T. Hirayamas and S. Fukuis, Appl. Organometall. Chem., 1988, 2, 339.

11 S. X. C. Le, W. R. Cullen and K. J. Reimer, Environ. Sci. Technol., 1994, 28, 1598.

12 W. R. Chappell and C. O. Abernathy, in Arsenic Exposure and Health, ed. W. R. Chappell, C. O. Abernathy and C. R. Cothern, Science and Technology Letters, Northwood, Middlesex, UK, 1994, ch. 2, pp. 21-29.

13 G. Lunde, J. Sci. Food Agric., 1973, 24, 1021

14 Toxicological Profile for Arsenic, ATSDR/TP-88/02, prepared by Life Systems Inc. for Agency for Toxic Substances and Disease Registry U.S. Public Health Service, in collaboration with US EPA, March 1998, Oak Ridge National Laboratory, TN, USA, 1989.

15 H. Hasegawa, Y. Sohrin, M. Matsui, M. Hojo and M. Kawashima, Anal. Chem., 1994, 66, 3247.

16 S. J. Haswell, P. O’Neill and K. C. C. Bancroft, Talanta, 1985, 32, 69.

17 C. Hwang and S. Jiang, Anal. Chim. Acta, 1994, 289, 205.

18 M. L. Magnuson, J. T. Creed and C. A. Brockhoff, J. Anal. At. Spectrom., 1996, 11, 893.

19 P. Thomas and K. Sniatecki, J. Anal. At. Spectrom., 1995, 10, 615

20 X. C. Le and M. Ma, Anal. Chem., 1998, 70, 1926.

21 M. Ma and X. C. Le, Clin. Chem., 1998, 44, 539.

22 X. Le, W. R. Cullen and K. J. Reimer, Clin. Chem., 1994, 40, 617

23 G. K. C. Low, G. E. Batley and S. J. Buchanan, Chromatographia, 1986, 22, 292.

24 E. H. Larsen, G. Pritzi and S. H. Hansen, J. Anal. At. Spectrom., 1993, 8, 557.

25 D. Heitkemper, J. Creed, J. Caruso and F. L. Fricke, J. Anal. At Spectrom., 1989, 4, 279.

26 G. M. Momplaisir, J. S. Blais, M. Quinteiro and W. D. Marshall, J. Agric. Food Chem., 1991, 39, 1448

27 E. A. Crecelius, Environ. Health Perspect., 1977, 19, 147.

28 R. Cornelis and J. De Kimpe, J. Anal. At. Spectrom., 1994, 9, 945.

29 B. S. Sheppard, J. A. Caruso, D. T. Heitkemper and K. A. Wolnik, Analyst, 1992, 117, 971.

30 J. P. Buchet, J. Pauwels and R. Lauwerys, Environ. Res., 1994, 66 , 44.

31 K. D. Arbinda, R. Chakraborty, M. L. Cervera and M. de la Guardia, Mikrochim. Acta, 1996, 122, 209

32 W. C. Story, J. A. Caruso, D. T. Heitkemper and L. Perkins, J. Chromatogr. Sci., 1992, 30, 427.

33 J. Alberti, R. Rubio and G. Rauret, Fresenius' J. Anal. Chem., $1995,351,420$.

34 D. Beauchemim, K. W. M. Siu, J. W. McLaren and S. S. Berman, J. Anal. At. Spectrom., 1989, 4, 285.

35 E. H. Larsen, G. Pritzi and S. H. Hansen, J. Anal. At. Spectrom., $1993,8,1075$.

36 M. A. Lopez, M. M. Gomez, M. A. Palacios and C. Camara, Fresenius' J. Anal. Chem., 1993, 346, 643.

37 M. Van Holderbeke, Y. Zhao, F. Vanhaecke, L. Moens, R. Dams and P. Sandra, J. Anal. At. Spectrom., 1999, 14, 229.

38 B. Michalke and P. Schramel, Electrophoresis, 1998, 19, 2220.

39 S. A. Pergantis, K. A. Francesconi, W. Groessler and J. E. Thomas-Oates, Anal. Chem., 1997, 69, 4931.

40 K. W. M. Siu, G. J. Gardner and S. S. Berman, Rapid Commun Mass Spectrom., 1988, 2, 69.

41 J. J. Corr and E. H. Larsen, J. Anal. At. Spectrom., 1996, 11, 1215.

42 S. A. Pergantis, W. Winnik and D. Betowski, J. Anal. At. Spectrom., 1997, 12, 531.

43 K. W. M. Siu, R. Guevremont, J. C. Y. LeBlanc, G. J. Gardner and S. S. Berman, J. Chromatogr., 1991, 554, 27.

44 X. C. Le, M. Ma and N. A. Wong, Anal. Chem., 1996, 68, 4501

45 R. H. Atallah and D. A. Kalman, Talanta, 1991, 38, 167.

46 J. S. Edmonds and K. A. Francesconi, Nature, 1981, 289, 602.

47 P. A. Gallagher, J. W. McKiernan, X. Wei, C. A. Brockhoff, J. A. Shoemaker, J. T. Creed and J. A. Caruso, European Winter Conference on Plasma Spectrochemistry, Pau, France, January 10-15, 1999, C7.

48 X. C. Le, W. R. Cullen and K. J. Reimer, Talanta, 1994, 41, 495ss.

Paper 9/06249A 